\title{
Enhancing Cell Block Quality- A Comparative Study of Formalin and Agar-Based Methods
}

\author{
Shruti Mishra ${ }^{1 *}$, Kishore Kumar $\mathrm{S}^{2}$. and Dipanwita $\mathrm{Nag}^{3}$ \\ ${ }^{1}$ Department of Pathology and Lab Medicine; All India Institute of Medical Sciences, Bhubaneswar, INDIA \\ ${ }^{2}$ Department of Hematology, MIOT International Hospital, Chennai , INDIA \\ ${ }^{3}$ Department of Pathology, Medical College, Kolkata, INDIA
}

\begin{abstract}
Background: There are not many studies conducted in India to compare cell block preparation methods with reagents and materials that are readily available in all laboratories. This study aimed to standardize and compare two simple cell block techniques, which can be done in low resource settings too.In the study, 35 cases of thyroid, lymph node, and breast were collected for both FNA and cell block preparation for six months.

Materials and Methods: There were separate passes given for both methods. A total of seventy cell blocks made using formalin and agar methods of preparation.

Results: We compared both the methods on technical and morphological levels. The formalin method was overall easy to perform and was yielding good morphological results in $98 \%$ cases, the only drawback being cell loss during handling and processing. While in the agar method, there was almost no cell loss, but it was more technically difficult and yielded poorer morphological results. A scoring system was made for cellularity: no cells $=0$, hypo-cellular $=1+$, hypo-cellular with tissue fragments $=2+$, cellular $=3+.18 \mathrm{~A}$ score of $2+$ and $3+$ was scored by $31 / 35$ formalin blocks and $28 / 35$ agar blocks.
\end{abstract}

Conclusions: The sensitivity of both formalin and agar methods are almost comparable. However, the procedure of the formalin method is far more straightforward and user friendly. Moreover, it also provides a better architectural picture than the agar method.

\section{Keywords: Cellblock, Formalin Method, Agar Method}

\section{Abbreviations: \\ CB: Cell Block \\ FNA: Fine-needle aspirate \\ CTC: CytoLyt-prefixed thrombin clot \\ IFS: Inverted filter sedimentation \\ PT: Plasma thromboplastin}

\section{Introduction}

Fine Needle Aspiration Cytology (FNAC) is an old, costeffective, and perhaps one of the best procedures for the initial evaluation and diagnosis of many lesions.[1,2] It is simple, safe, and reliable, primarily when performed under ultrasound guidance. Cellblock (CB) preparations are made by rinsing the aspirated material in cytology preservative solution after making the direct conventional smears. However, questions have been raised regarding both the cellular adequacy and consequently, the diagnostic accuracy of the CB method. $[3,4]$

Cellblock techniques are of value, especially to a pathologist. They somewhat resemble histological pattern in a cytology setting. [5, 6] Cell blocks provide an advantage of evaluating cytoarchitecture and micro-biopsies. Moreover, since we have the block further sections can be acquired which can be subjected to special stains and immunocytochemical stains too. $[7,8]$
This study aimed to compare two relatively simple methods of cellblock preparation in a limited resource setup. This study aimed to standardize and compare two simple cell block techniques, which can be done in low resource settings too. The objectives of our study were to compare the feasibility and results of the formalin and the agar methods, and to compare their sensitivity.

\section{Materials and Methods}

The study was conducted over six months. This study comprises of 15 thyroid cases, ten breast cases, and ten lymphadenopathy cases. A total of 70 cell blocks were prepared and compared with the histological findings of the same for surgery.

We can never compare apples with oranges. So, we made two separate passes for the two methods under comparison in each case. This process ruled out the bias of inadequate sampling in either of the methods. Material collected were stored in two different vials with buffered formalin and left for 24 hours. The next day, these samples were centrifuged for ten minutes at 2000rpm. The cell button obtained was removed from the test tube by a spatula. One cell button was wrapped in blotting paper and given for processing. The other cell button was accentuated 
by adding molten agar after properly draining off formalin. Then once it solidified, it was given for processing also.

The gold standard for comparison was the corresponding histology. The results were analyzed using standard statistical tools.

\section{Results}

Preparation of the Formalin block involved the least technical details. It was the simplest and the least time-consuming process and performed at room temperature. The Agar method was a more tedious process, as agar was initially converted and maintained in the liquid state. In cell blocks prepared with the Formalin method, cells tended to aggregate in the center of the clot and the cut sections. In contrast, in the Agar preparation, the cells gravitated to the bottom of the hardened pellet. This issue was addressed by dividing the Agar pellet in half and embedding the resulting hemispheres at the cut side.

The Agar method is useful in cases where aspirate was not enough to form a cell button of its own in the Formalin method. The formalin method also has more cell loss, unlike the Agar method. The formalin method was useful in thyroid cases where the vascularity of the organ leads to blood mixed aspirate. Here the blood clot itself formed a good base for holding the aspirated cells. [Table-1]
The cellularity of the Formalin method was good. While the Agar sections appeared to have low cellularity, with the cellular groups interspersed among large agar lakes. Between the two preparations, Formalin blocks revealed the best overall morphologic preservation with minimal artifacts. Cells in the Agar sections exhibited exaggerated cytoplasmic vacuoles, denser cytoplasm, and more frayed cytoplasmic borders. [Fig$1,2]$ However, proportions of cell blocks with excellent and fair morphologic preservation (defined as containing more and less than $50 \%$ of the cells of interests, respectively) were similar among both the methods. [Table-2]

Here we see that the Agar method has poor morphological preservation even though the cellularity was comparable with the Formalin method. The reason behind this was the splaying of the agar ribbons on the hot water bath.

\section{Discussion}

An ideal cell block should have the following features:

a) the cells should resemble corresponding cells in alcohol-fixed Papanicolaou (Pap)-stained smears, [9]

b) nuclear-cytoplasmic details should be well preserved,

c) easy recoverability and

Table 1: Comparison of technical aspects of Formalin and Agar Methods.

\begin{tabular}{|l|l|l|l|}
\hline SI. No & Technical Aspect & Formalin Method & Agar Method \\
\hline 1. & Requirement & $10 \%$ Formalin & $10 \%$ Formalin, Agar \\
\hline 2. & Technical intricacies & Simple & Difficult relative to Formalin method \\
\hline 3. & Time consumed & Less & More \\
\hline 4. & Storage requirement of reagents & Room temperature, no special need & Agar has to be stored in sterile environment. \\
\hline 5. & Form of use of reagent & Natural state & Agar has to be used in molten form \\
\hline 6. & Amount of reagent added & Doesn't make any difference & $\begin{array}{l}\text { Agar has to be added according to the volume of the aspirated } \\
\text { material, Dilution scare. }\end{array}$ \\
\hline 7. & Base for holding cells & Tissue fluid and blood & Agar \\
\hline 8. & Scanty aspirate & Less useful & More useful \\
\hline 9. & Pellet formed & Cells aggregates in the centre & Cells aggregate in the bottom \\
\hline 10. & Processing & Normal method & Normal method \\
\hline 11. & Loss of cell during processing & More & Less \\
\hline 12. & Blocking & Chance of cell loss more & Chance of cell loss less \\
\hline 13. & Microtome & Easy to cut thinner sections & Not easy to cut thinner sections \\
\hline 14. & Ribbon & Floats easily & Spreads out on hot water bath \\
\hline 15. & Staining & Takes good stain & Is a bit lighter \\
\hline
\end{tabular}

Table 2: Comparison of Morphological Appearance of Formalin and Agar Methods.

\begin{tabular}{|c|c|c|c|c|c|c|c|c|}
\hline \multirow[t]{2}{*}{ Method } & \multirow[t]{2}{*}{ Site } & \multicolumn{2}{|l|}{ Cellularity } & \multicolumn{2}{|c|}{ Cellular preservation } & \multicolumn{2}{|c|}{ Morphological preservation } & \multirow[t]{2}{*}{ Artefact } \\
\hline & & Moderate / High & Low & Good & Fair & Good & Fair & \\
\hline \multirow[t]{3}{*}{ Formalin } & \multirow{3}{*}{$\begin{array}{l}\text { Thyroid } \\
\text { Lymph nodes } \\
\text { Breast }\end{array}$} & $94 \%$ & $6 \%$ & $94 \%$ & $6 \%$ & $94 \%$ & $6 \%$ & \multirow[t]{3}{*}{ Present } \\
\hline & & $90 \%$ & $10 \%$ & $100 \%$ & 0 & $100 \%$ & 0 & \\
\hline & & $80 \%$ & $20 \%$ & $90 \%$ & $10 \%$ & $100 \%$ & 0 & \\
\hline \multirow[t]{3}{*}{ Agar } & \multirow{3}{*}{$\begin{array}{l}\text { Thyroid } \\
\text { Lymph nodes } \\
\text { Breast }\end{array}$} & $74 \%$ & $26 \%$ & $94 \%$ & $6 \%$ & $60 \%$ & $40 \%$ & \multirow[t]{3}{*}{ Present } \\
\hline & & $80 \%$ & $20 \%$ & $90 \%$ & $10 \%$ & $70 \%$ & $30 \%$ & \\
\hline & & $90 \%$ & $10 \%$ & $90 \%$ & $10 \%$ & $60 \%$ & $40 \%$ & \\
\hline
\end{tabular}


Table 3-Sensitivity of Cell Blocks

\begin{tabular}{|c|c|c|c|c|}
\hline SI.No. & Site & HPE & Formalin Method & Agar Method \\
\hline \multirow[t]{3}{*}{1.} & \multirow{3}{*}{$\begin{array}{l}\text { Thyroid Malignant } \\
\text { Benign Inconclusive }\end{array}$} & $34 \%$ & $34 \%$ & $14 \%$ \\
\hline & & $66 \%$ & $60 \%$ & $60 \%$ \\
\hline & & & $6 \%$ & $26 \%$ \\
\hline \multirow[t]{3}{*}{2.} & \multirow{3}{*}{$\begin{array}{l}\text { Lymph node Malignant } \\
\text { Benign Inconclusive }\end{array}$} & $40 \%$ & $40 \%$ & $30 \%$ \\
\hline & & $60 \%$ & $50 \%$ & $50 \%$ \\
\hline & & & $10 \%$ & $20 \%$ \\
\hline \multirow[t]{3}{*}{3.} & \multirow{3}{*}{$\begin{array}{l}\text { Breast Malignant } \\
\text { Benign Inconclusive }\end{array}$} & $70 \%$ & $60 \%$ & $70 \%$ \\
\hline & & $30 \%$ & $20 \%$ & $20 \%$ \\
\hline & & & $20 \%$ & $10 \%$ \\
\hline
\end{tabular}

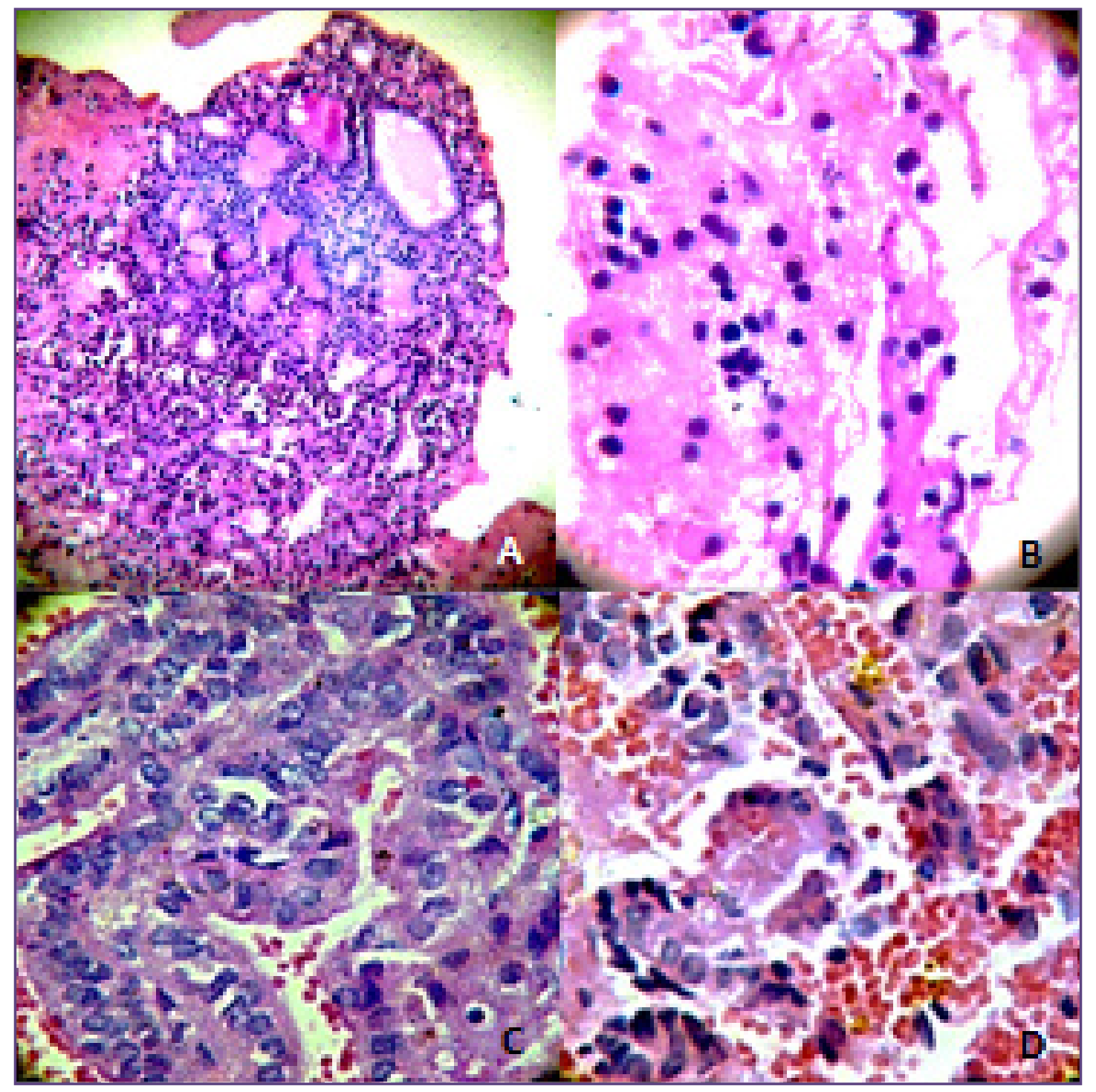

Fig. 1: a] H\&E 100x- Picture shows features of colloid goitre with follicles of varied sizes in formalin block., b] H\&E 200xPicture shows scattered follicular cells in agar block of the same case., c] H\&E 400x- Picture shows features of papillary carcinoma of thyroid with the characteristic nuclear features in formalin block.m d] H\&E 400x- Picture shows fragmented papillae in agar preparation of the same case. 


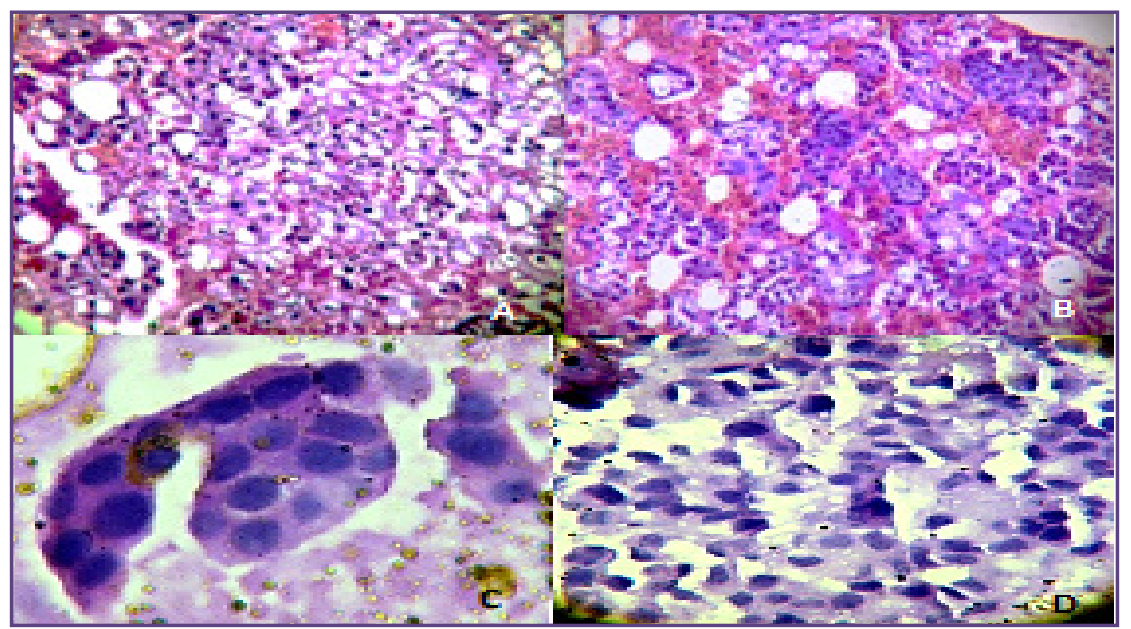

Fig. 2: a] H\&E 100x- Picture shows adenocarcinoma deposits in lymph node in formalin block., b] H\&E 100x- Picture shows same deposits in agar block also but the cells are not compact., c] H\&E 400x- Picture shows features of ductal carcinoma in formalin block.. d] H\&E 400x- Same case in agar block with better cellularity.

d) cellularity and cellular characteristics should be well maintained to render them suitable for ancillary studies such as special stains and immune-staining.

An ideal method for cell block preparation would be both simple and reproducible in routine settings of all laboratories. [9] It has been proved by many studies that CB prepared from FNA, and fluid samples have got diagnostic significance. It provides not only cyto-architectural pictures but also gives immunocytochemical information too. [10-13] There are several time-proven methods of cell block preparation. Few studies have compared the performance of different cell block methodologies. [7,14,15]

Jing et al. [16], in their study, found out that the CytoLytprefixed thrombin clot (CTC) method gave more or less same results as plasma-thrombin and histogel method. Nigro et al. [17], in their study, compared four methods. They found that the plasma thrombin method was the best among all methods. Other methods compared were inverted filter sedimentation (IFS), albumin method, simple sedimentation.

Out of all, PT (Plasma Thromboplastin) method is the most popular. This method was not used in our study because firstly, it needs pooled plasma, which is not readily available in our setup, and secondly, thromboplastin reagent is costly and needs proper storage facility. As there is hardly any study of method comparison in our country, we have conducted this study to find which method of cell block preparation can be done without much hassle in every kind of setups.

The methods used in our study were the Formalin method and the Agar method. Formalin method requires washing of the hub of the syringe with formalin followed by 24 hours fixation. The next day it was centrifuged, and the pellet was wrapped in blotting paper and given for processing. The shortcomings in the technical aspects of this method were the loss of cells in various steps of processing and blocking. Moreover, cell poor aspirates were found to be unfit for processing; that is, the button was too small to be retrieved from the vial.

In the Agar method, formalin was utterly drained and dried from the cell button before molten agar was added. Once it solidified, the button was retrieved and wrapped in a blotting paper and given for processing as usual. The pitfalls in this method were quite a few. First of all, agar being a culture media, had to be stored in a sterile environment. It required agar to be melted and maintained at this molten state for proper mixing, which was quite a tedious job. Most importantly, care must be taken for the amount of agar added should not exceed the volume of tissue; otherwise, it would dilute the aspirated material. The cost of this method is also more than the Formalin method. [Table-1]

Agar method posed difficulty during microtomy as the ribbons tend to splay when placed on the hot water bath, and it was also not easy to get thinner sections. [Fig-1,2] Staining was also lighter in agar sections. These few drawbacks resulted in a mild visual haziness in comparison to the formalin method.

Out of fifteen thyroid cases, five were malignant, and the rest were benign. [Fig-1] Four lymph node cases were malignant, and six were benign. [Fig-2] Lastly, seven breast cases were malignant, and the rest three benign. [Fig-2]

Adequate cellularity was obtained in $88 \%$ formalin blocks, and $82 \%$ agar blocks. The formalin method shows a median of $90 \%$ against a median of $80 \%$ in the agar method. A scoring system was made for cellularity: no cells $=0$, hypo-cellular $=1+$, hypo-cellular with tissue fragments $=2+$, cellular $=$ $3+$. [17] 31/35 formalin blocks and 28/35 agar blocks had a score of $2+$ to $3+$. Preservation of cellularity was almost comparable in both methods. The median for the formalin method was $94 \%$, and the agar method was $90 \%$. However, 
to our surprise, morphology was much better preserved in the Formalin method than the Agar method. The median for good morphological preservation in the Formalin method was $92 \%$, while the median in the Agar method it was $62 \%$.

Sensitivity was calculated by comparing the results of cellblock reports and the final histopathology reports. Sensitivity by formalin method for malignant thyroid diagnosis was $100 \%$ in comparison to $45 \%$ in the agar method. The sensitivity for benign thyroid lesion detection was $94 \%$ in both cases. In the case of malignant lymph node, the formalin method had a sensitivity of $100 \%$ and $75 \%$ in the agar method. However, in malignant breast cases, the formalin method has a sensitivity of $86 \%$, while the agar method had $100 \%$ sensitivity. [Table-3]

\section{Conclusion}

Finally, we see that the sensitivity of both formalin and agar methods are almost comparable. Nevertheless, the procedure of the formalin method is far more straightforward and user friendly. Moreover, it also provides a better architectural picture than the agar method.

\section{Acknowledgment}

We would like to acknowledge the support of the technical staff.

\section{Funding}

None

\section{Competing Interests}

None declared

\section{References}

1. Nguyen GK, Lee MW, Ginsberg J, Wragg T, Bilodeau D. Fineneedle aspiration of the thyroid: an overview. Cyto Journal. $2005 ; 2: 12$.

2. Deveci MS, Deveci G, LiVolsi VA, Baloch ZW. Fine-needle aspiration of follicular lesions of the thyroid. Diagnosis and follow- up. Cyto Journal. 2006; 3:9.

3. Sanchez N, Selvaggi SM. Utility of cell blocks in the diagnosis of thyroid aspirates. Diagn Cytopathol. 2006, 34:89-92.

4. Chen PK. Artifacts of cytology cell block in fine-needle aspiration biopsy of thyroid. Diagn Cytopathol. 2004, 31:362-3.

5. Shivakumarswamy U, Arakeri SU, Karigowdar MH, Yelikar B. Diagnostic utility of the cell block method versus the conventional smear study in pleural fluid cytology. J Cytol. $2012 ; 29: 11-5$.
6. Rowe LR, Marshall CJ, Bentz JS. Cell block preparation as an adjunctive diagnostic technique in ThinPrep monolayer preparations: a case report. Diagn Cytopathol. 2001; 24:142-4.

7. Akalin A, Lu D, Woda B, Moss L, Fischer A. Rapid cell blocks improve accuracy of breast FNAs beyond that provided by conventional cell blocks regardless of immediate adequacy evaluation. Diagn Cytopathol. 2008; 36:523-9.

8. Sethi S, Geng L, Shidham VB, Archuletta P, Bandyophadhyay $\mathrm{S}$, Feng J, et al. Dual-color multiplex TTF-1 + napsin A and p63 + CK5 immunostaining for subcategorizing of poorly differentiated pulmonary non-small carcinomas into adenocarcinoma and squamous cell carcinoma in fine-needle aspiration specimens. Cyto Journal. 2012; 9:10.

9. Nathan NA, Narayan E, Smith MM, Horn MJ. Cellblock cytology: improved preparation and its efficacy in diagnostic cytology. Am J Clin Pathol. 2000; 114:599-6.

10. Kulkarni MB, Desai SB, Ajit D, Chinoy RF. Utility of the thromboplastin-plasma cellblock technique for fine-needle aspiration and serous effusions. Diagn Cytopathol. 2009; 37: 86-90.

11. Fetsch PA, Simsir A, Brosky K, Abati A. Comparison of three commonly used cytologic preparations in effusion immunocytochemistry. Diagn Cytopathol. 2002; 26:61-6.

12. Yang GC, Wan LS, Papellas J, Waisman J. Compact cell blocks: use for body fluids, fine needle aspirations and endometrial brush biopsies. Acta Cytol. 1998; 42:703-6.

13. Smedts F, Schrik M, Horn T, Hopman AH. Diagnostic value of processing cytologic aspirates of renal tumors in agar cell (tissue) blocks. Acta Cytol. 2010; 54:587-94.

14. Gorman BK, Kosarac O, Chakraborty S, Schwartz MR, Mody DR. Comparison of breast carcinoma prognostic/predictive biomarkers on cell blocks obtained by various methods: Cellient, formalin, and thrombin. Acta Cytol. 2012; 56:289-96.

15. Wagner DG, Russell DK, Benson JM, Schneider AE, Hoda RS, Bonfiglio TA. Cellient automated cell block versus traditional cell block preparation: a comparison of morphologic features and immunohistochemical staining. Diagn Cytopathol. 2011; 39:730-36.

16. Jing X, Li QK, Bedrossian U, and Michael CW. Morphologic and Immunocytochemical Performances of Effusion Cell Blocks Prepared Using 3 Different Methods. Am J Clin Pathol. 2013; 139:177-82.

17. Nigro K1, Tynski Z, Wasman J, Abdul-Karim F, Wang N. Comparison of cell block preparation methods for nongynecologic ThinPrep specimens. Diagn Cytopathol. 2007; 35:640-3.

*Corresponding author:

Dr. Shruti Mishra, Postal Address: Plot no. 521, Saheed Nagar, Bhubaneswar- 751007 INDIA

Phone: +91 8017377253

Email: msshruti6@gmail.com

Date of Submission : 27/11/2019

Financial or other Competing Interests: None. 